\title{
Pricing of Virtual Goods and Designing Game Challenge Level for Free-to- Play Mobile Games in the Presence of Copycat Competitors
}

\author{
Ismail Civelek \\ Western Kentucky University \\ ismail.civelek@,wku.edu
}

\author{
Yipeng Liu \\ Northern Illinois University \\ yliu@niu.edu
}

\author{
Sean R. Marston \\ Western Kentucky University \\ sean.marston@,wku.edu
}

\begin{abstract}
In-game purchases, virtual currency, content design for heterogeneous consumers and strong competition are key challenges for mobile game providers. This study addresses determination of optimal game design strategies for game providers in the presence of heterogeneous players and copycat competitors. Moreover, this paper incorporates pricing of virtual goods/currency into the Free-toPlay $(F 2 P)$ mobile game design via a duopoly model and characterizes the optimal strategies for game providers in terms of pricing of virtual goods/currency and the game challenge level.
\end{abstract}

\section{Introduction}

The digital game industry is growing at a very fast rate bringing in $\$ 83.6$ Billion in 2014 and was expected to increase by $19.1 \%$ to $\$ 99.6$ Billion in 2016 [6]. The way digital games are played has evolved during the past few years as the sales of mobile devices have increased dramatically. While PC gaming and console gaming are still leaders in the way that digital games are played, the mobile gaming segment has been steadily increasing with 966 million players worldwide [12], bringing in revenue of $\$ 34.8$ billion. Top mobile games can be very successful in generating significant revenue, Supercells games' Clash of Clans and Hay Day generated $\$ 2.4$ million a day [17]. The dominant way for mobile games to earn revenue has been shifting from the traditional pay outright for the game to a free to play model, which earns revenue through micro transactions.

The main difference between mobile F2P and console games is that there is no free trial for players; instead players download the entire game for free [3]. Thus, there are no barriers for players to download and start playing an F2P mobile game. Players can still play the entire game without spending real money; however, many players are willing to buy virtual goods/currency to speed up their game progression. In today's mobile game market, F2P game developers prefer as many players as possible present in the game due to network effect, because most F2P games are massively multiplayer online games. Therefore, these mobile games are totally free to download without any free-trial period.

This study focuses on F2P mobile games generating revenue from selling virtual currency (i.e. diamonds, crystals that can be used to purchase armor, equipment or faster leveling in game) or virtual goods. In-app purchases will be the number one revenue source for mobile apps while paid apps will account for $37.8 \%$ and ad based revenue will account for $14 \%$ at the end of 2017 [11]. Therefore, our study only focuses on revenue generated by selling virtual currency/goods in F2P mobile games. This study's main contribution is providing a framework for a game provider facing strong competition and heterogeneous players.

This study incorporates pricing of virtual goods/currency into the F2P mobile game design via a duopoly model and characterizes the optimal strategies for game providers in terms of pricing of virtual goods/currency and the game challenge level. The purpose of our monopoly and duopoly models is to explore strategies and provide managerial insights for the original game providers in pricing of virtual goods and setting challenge level of the F2P mobile games. In the duopoly model, we investigate a commonly observed practice, where the copycat game providers compete against the original game providers through duplicating the game mechanism of the original game. We reveal the optimal pricing and game design strategies for both the original and copycat game providers engaged in a duopoly competition. In addition, we show that there should be a reasonable first-mover advantage for the original game provider to create original games.

We review the literature in Section 2; then, our modeling approach and results are presented in Sections 3 . We conclude our study with a discussion, managerial implications for game providers and future research directions in Section 4. 


\section{Literature Review}

Regarding current research in digital games, most of the literature focuses on traditional computer and console games, studies embracing virtual currency, game design and competition among F2P game providers are very limited. The goal of this literature review is to show that this paper fills a gap in mobile game (specifically F2P games) design incorporating pricing of virtual goods/currency for both monopoly and duopoly cases. In a recent article, Guo et al. [7] study a monopoly game provider's problem of selling virtual currency to players who enjoy leisure and earn virtual currency. They conclude that decreasing the virtual currency price and increasing the number of virtual goods would improve game providers' revenue. Finneran and Zhang [6] provide a review of promises and challenges of studying flow, a psychological state, in computer-mediated environments and cautions researchers to investigate hidden assumptions of theories in other disciplines

Agarwal and Karahanna [1] use a structural equation analysis to examine cognitive absorption of information technology use based on temporal dissociation, focused immersion, heightened enjoyment, control, and curiosity. They propose that playfulness and personal innovativeness are key determinates of cognitive absorption. Liu et al. [13] argue that competition is the key factor of game design that should be incorporated into organizational activity games such as employee training games.

This paper only focuses on F2P games on mobile devices. Regarding game satisfaction and virtual currency in F2P games, researchers have been studying motivations of playing the games and impacts of virtual currency and promotions. Yee [18] presents that achievement, social environment and immersion components are main reasons for playing video games. Moreover, Ryan et al. [15] demonstrate that game enjoyment; autonomy, competence and relatedness are important factors for intentions to play video games. Besides players' intentions to play these video games, Moon et al. [14] propose ownership-enhancing and socialization-enhancing strategies to improve player commitment in playing the game.

Considering virtual currency and promotions in video games, Guo and Barnes [8] model consumer's behavior buying virtual currency via a mixture of new constructs and established theories, including theory of planned behavior, technology acceptance model, trust theory and unified theory of acceptance and use of technology. Additionally, Hamari and Lehdonvirta [10] focus on marketing of virtual goods for F2Ps due to untapped potential for marketing of virtual goods and Hamari [9] investigates purchase behavior for virtual goods in three F2P game environments: social networking, first-person shooter and social virtual world games.

This paper examines micro transactions in digital games, specifically in F2P mobile games. This study is positioned in the interface of information systems, e-commerce, and economics literature. This study also characterizes the optimal strategies for game providers in terms of pricing of virtual goods/currency, game challenge level and threat from copycat game providers.

\section{Duopoly Model}

Cloning is as old as the video game industry itself. In the mobile game market, the problem of copycat games is an even more staggering problem due to familiarity with the popular games and an increased chance of capturing the attention of players (e.g., Pokémon Go vs. Citymon Go, Clash of Clans vs. Game of War, Super Mario Bros vs Super Max, 2048 vs. Threes etc.) [2]. With a seemingly infinite number of games on mobile platforms, it is quite common to see many games which are extremely similar to each other, particularly when you consider the features of the gameplay mechanics. Analyzing a duopoly case in our problem coincides with the copycat problem of the F2P mobile game market. In our model, there are two game providers, A and B, whose F2P games are competing for the same market. Thus, the game providers need to make their decisions not only to capture market share and generate revenue from players buying virtual goods/currency, but also to consider the strategy of the other game providers.

In mobile games, we observe that the amount of virtual goods purchased by consumers vary from player to player. To model the "free to play" virtual game scenario, we first require that consumers be heterogeneous in their gaming challenge level preferences. Let $\theta$ represents individual player's preference about the challenge level of the game, which is assumed to be distributed uniformly between 0 and 1 . Hence, the population density is normalized at 1 . Players are heterogeneous in $\theta$, this follows from the classic Shapiro's [16] treatment of consumers' heterogeneous tastes for product qualities. Players may consume different amount of virtual goods/currency depending on the actual game challenge level relative to individual player's personal preference. This is also the departing point for our model from the existing literature (e.g., [7, $8]$ ), where players are often assumed to consume 
either zero or one quantity of the game's virtual goods/currency. A mobile game player may not play the game at all if the game is deemed as too difficult for a beginner or too easy for a more experienced player. Hence, the actual game challenge level has an impact on the potential market size for the game. Therefore, we treat the actual game challenge level $\gamma$ as one of the two decision variables of the game providers in addition to the unit price $\mathrm{p}$ for the virtual goods/currency.

The game challenge level will cause disutility to a player in both directions when the game challenge level set by the provider and player's preference on the game challenge level are not equal to each other. When a game fails to meet a player's preconception, either by being too easy or too challenging for that player, it often causes frustration to the player's game experience. Lowering the challenge level to "Easy" can feel humiliating to a self-titled "hardcore" player, as raising the challenge level to "Hard" would be unthinkable to a "casual" one. When the game challenge level is higher than a player's preferred challenge level, the player may choose to purchase a certain amount of virtual goods/currency to align the game's challenge level with the player's preferred challenge level and therefore improve the player's utility. Meanwhile, if the game challenge level is lower than a player's preferred challenge level, then the player will not purchase any virtual goods/currency as doing so will only decrease a player's utility.

Without loss of generality, we assume game provider $B$ creates a copycat game of provider A; thus, the problem is modeled as a three-stage Stackelberg game. In Stage 1, the original game provider $A$ announces the game challenge level $\gamma_{A}$ and the unit price for the virtual goods/currency $p_{A}$ for its game. In stage 2 , the copycat announces game challenge level $\gamma_{B}$ and the unit price for the virtual goods/currency $p_{B}$ for its game observing the strategies adopted by provider $A$. In stage 3 , players decide which game to play and choose the amount of virtual goods/currency $G_{A}$ and $G_{B}$ to purchase. The duopoly case is solved by backward induction.

\subsection{Player's Decision in Stage 3}

By backward induction, first we solve the player's decision problem in Stage 3 assuming the game challenge levels (both original and copycat games) and the unit prices for the virtual goods/currency are already observed by players. A player's utility function has the following forms depending on the choice of the F2P game, $U_{A}=V-c\left(\gamma_{A}-\theta-G_{A}\right)^{2}-p_{A} G_{A}$ or
$U_{B}=V-s-c\left(\gamma_{B}-\theta-G_{B}\right)^{2}-p_{B} G_{B}$, as $s$ is the discounted utility for the copycat game.

We assume a penalty $s>0$ for the gross utility of the copycat game. This is because the original game often offers a larger player base and is deemed by players as more valuable due to positive network effects. Furthermore, most F2P mobile games are multiplayer, there are already more players playing the original game when the copycat game is introduced. Hence, the copycat game provider will suffer for not being first to market. In our analysis, we also choose to focus on the duopoly competition between the original game and the copycat when the market is fully covered. An original game with large gross utility often enjoys an initial release success, which not only attracts a lot of players but also draws copycat competitors due to its popularity. Hence, in F2P games if an original game is indeed very popular then a copycat game is almost guaranteed to show up, hence a duopoly competitor.

Since $U_{A}$ and $U_{B}$ are concave in $G_{A}$ and $G_{B}$ respectively, the optimal amount of virtual goods/currency purchased by the player is found by solving the first order conditions and the optimal solutions are $G_{A}{ }^{*}=\gamma_{A}-\theta-\left(p_{A} / 2 c\right)$ and $G_{B}{ }^{*}=\gamma_{B}-\theta-\left(p_{B} / 2 c\right)$. Setting $G_{A}{ }^{*}=0$ and $G_{B}{ }^{*}=0$, we find the indifference points $\left(\theta_{U A}\right.$ and $\left.\theta_{U B}\right)$ for players purchasing virtual goods/currency as $\theta_{U A}=\gamma_{A^{-}}\left(p_{A} / 2 c\right)$ and $\theta_{U B}=\gamma_{B^{-}}\left(p_{B} / 2 c\right)$.

\subsection{Copycat's Decision in Stage 2}

The copycat game provider's objective is to optimize its revenue using both its game's challenge level and the pricing of the game's virtual goods/currency. If the copycat provider sets the unit price for its virtual goods higher than the original game provider, then the duopoly game is expected to be dominated by the original game provider. This is because players already perceive the copycat game as the less valuable product (due to the discount factor s), if the virtual goods in copycat game are more expensive, players would have no incentive to play the copycat game. Therefore, we choose to focus on the case where $p_{B} \leq p_{A}$.

Considering to ensure that the copycat game provider's revenue is positive, there exists an upper bound such that $p_{B}<2 c \gamma_{B}-\sqrt{ }\left(p_{A}{ }^{2}-4 c p_{A} \gamma_{A}+4 c\left(s+c \gamma_{B}{ }^{2}\right)\right)$. Note that this upper bound on $p_{B}$ is smaller than the lower bound on $p_{A}$. Therefore, the price set on the virtual goods/currency sold by the original game provider is always going to be greater than the copycat game provider i.e., $p_{A}>p_{B}$, which is consistent with our previous assumption. The copycat game must offer virtual goods/currency at a cheaper price than the original game to have positive revenue. 
Failing to provide cheaper virtual goods/currency will result in players choosing to participate only in the original game.

According to our analysis, the optimal price and challenge level for the copycat provider depends on the original game provider's challenge level and price. The copycat provider's optimal price will be lower than the original game provider's price; hence, this will allow the copycat to attract players. There exists an optimal game challenge level for the copycat provider in response to the original game providers' strategies set in Stage 1.

\subsection{Original Game Provider's Decision in Stage 1}

In Stage 1, the original game provider $A$ sets its price and challenge level with the expectation that a copycat game will show up in Stage 2. The revenue function of the original game provider is derived based on the targeted market between $\left[\theta_{i}, \theta_{U A}\right]$. The original game provider's optimal $p_{A}$ and $\gamma_{A}$ can be found by substituting the optimal $p_{B}{ }^{*}$ and $\gamma_{B}{ }^{*}$ (found in Stage 2) into $R_{A}$ and then we solve the revenue maximization problem for the original game provider.

The upper bound of $p_{A}$ is derived based on the constraint that $0<\theta_{i}<1$ and the lower bound of $p_{A}$ is derived based on the constraint that $\theta_{U A}>\theta_{i}$. Solving the above maximization problem, we obtain the optimal unit price for the original game provider such that $p_{A}{ }^{*}=(2 / 15)\left(7 c-2 \sqrt{ }\left(c^{2}+15 c s\right)\right)$. Since the revenue function $R_{A}$ is convex in $\gamma_{A}$, the interior optimal solution of $\gamma_{A}{ }^{*}$ do not exist. As a result, we analyze the optimal $\gamma_{A}{ }^{*}$ as well as the optimal unit price $p_{A}{ }^{*}$ numerically.

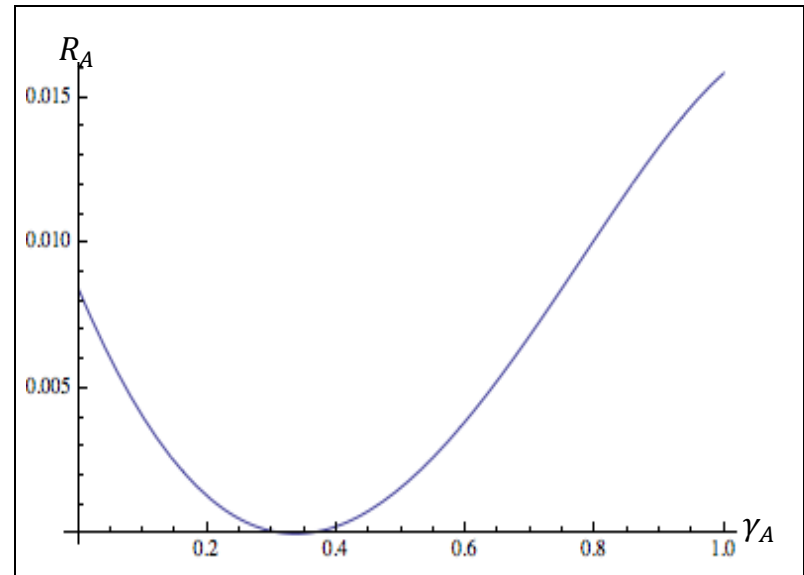

Figure 1. The Change of the Orginal Game Provider's Revenue Function with respect to $\gamma_{\mathrm{A}}$

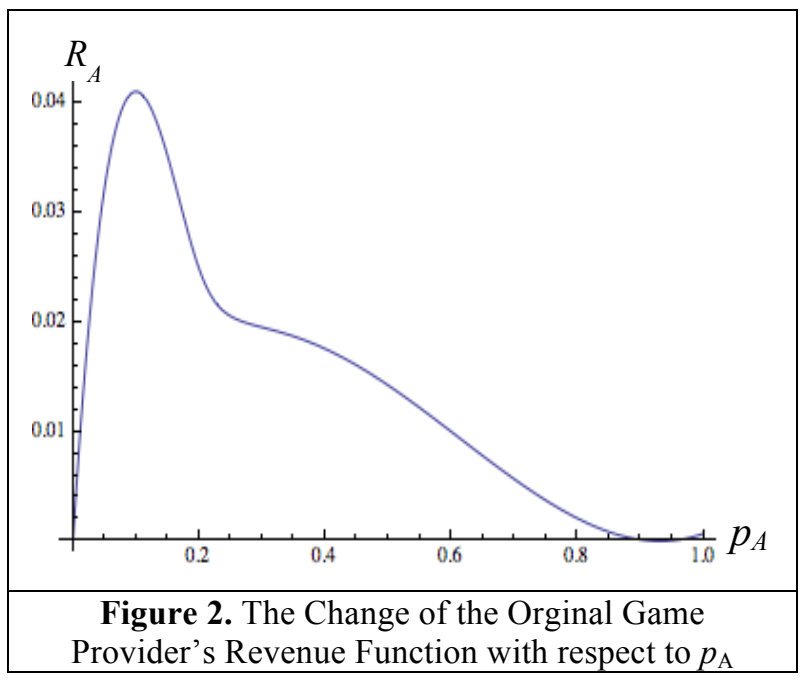

Figures 1 and 2 illustrate the change of the original game provider's revenue function with respect to $\gamma_{\mathrm{A}}$ and $\mathrm{p}_{\mathrm{A}}$. We set $\mathrm{c}=0.35, \mathrm{p}_{\mathrm{A}}=0.11 \mathrm{~s}=0.1$ in Figure 1 and $\mathrm{c}=0.35, \gamma_{\mathrm{A}}=1, \mathrm{~s}=0.1$ in Figure 2. The revenue function $R_{A}$ as shown in Figure 1 is convex in $\gamma_{\mathrm{A}}$. Starting approximately at $\gamma_{\mathrm{A}}=0.33$, the original game provider may choose to raise the game challenge level to increase its overall revenue. Moreover, raising the game challenge level to the highest value leads to the possible maximized revenue. This indicates the optimal solution for the game challenge level is found at $\gamma_{\mathrm{A}}{ }^{*}=1$. In another word, it is in the original game provider's best interest to offer the most challenging game in the duopoly setting. Furthermore, if the original game provider does not set $\gamma_{\mathrm{A}}$ to the upper bound value, the copycat provider will steal market share by cutting the price of its virtual goods/currency. That is not a desirable situation for the original game provider; thus, the challenge level $\gamma_{\mathrm{A}}$ should be the maximum feasible value based on previously set parameters. Also illustrated in Figure 2, although the revenue function $R_{A}$ is not strictly concave in $p_{A}$, the optimal value for the unit price $p_{A}$ that maximizes the original game provider's revenue does exist (approximately at $\left.\mathrm{p}_{\mathrm{A}}=0.11\right)$ in the shown example, which is consistent with the closed form solution presented previously, $p_{A}{ }^{*}=(2 / 15)\left(7 c-2 \sqrt{ }\left(c^{2}+15 c s\right)\right)$.

\subsection{Impact of First-Mover Advantage, $s$}

Figure 3 illustrates the impact of the discount factor $\mathrm{s}$ on the revenues of the two game providers. In Figure 3, we set $\mathrm{c}=0.35, \mathrm{p}_{\mathrm{A}}=0.11$ and $\gamma_{\mathrm{A}}=1$, the red line represents the original game provider's revenue and the black line represents the copycat game provider's revenue. 


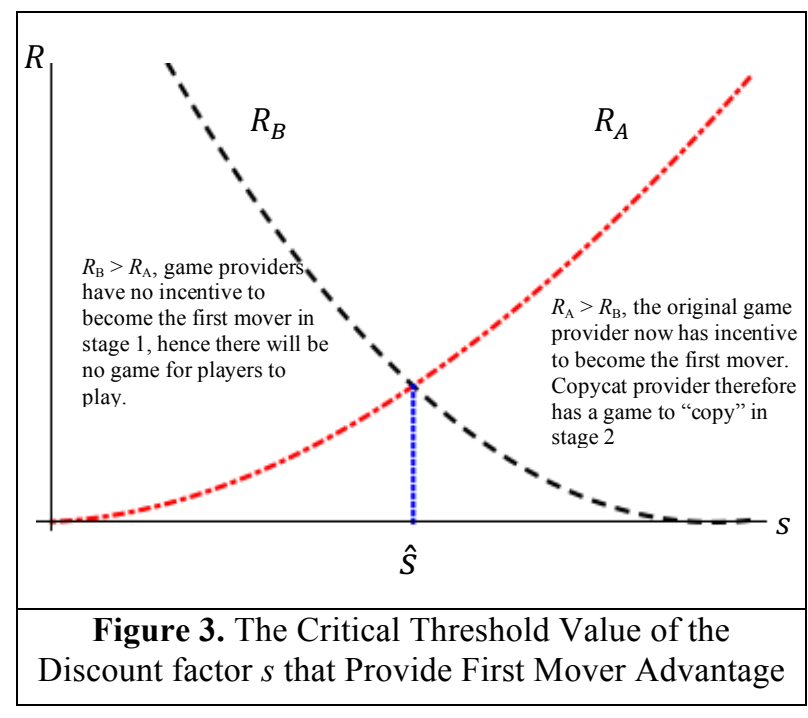

As shown in Figure 3, when the first-mover advantage is small, the revenue of the copycat game provider's revenue is higher than the original game provider. If the discount factor value is known to the original game provider beforehand, then the original game provider is better off as a copycat. Thus, no original game would be introduced to the market at all. The discount factor or penalty $s$ reflects a firstmover advantage for the original game provider. To ensure that there is a healthy market in which original games will continue to be produced, the industry can examine the use of regulations. For example, by regulating the release time of games, the industry can potentially ensure that a reasonable high first-mover advantage and prevent market failure.

While this study does not recommend regulation, it's potentially in the industries' best interest to examine possible use of regulations to sustain a healthy mobile game market. The larger the first mover advantage the more incentive for the original game provider to develop brand new games. The original game provider may pursue legal methods through copyright or patent protection to secure a penalty from the copycat provider so that the discount factor is big enough to provide necessary incentives. But often, the copycat game only clones the gameplay and mechanics of the original game, which is not copyrightable or enforceable. Thus, the original game provider should consider investing more in the content of the game so that it will take longer and make it harder for the copycat provider to clone. By releasing the game first into the market, the original game provider also enjoys the first mover advantage in building up its play base. In choosing F2P mobile games, players often favor the game with bigger player base. From the copycat game providers' perspective, it is in their best interest to reduce the first mover advantage received by the original game provider. Copycat game provider may achieve this by releasing the cloning version of the game soon after the release of the original game before the original game builds up dominated player base. This practice is often observed in real world, when a popular mobile game is released a copycat game tends to follow quickly. For example, Clash of Clans was released in August 2012 and less than a year later Game of War was released in July 2013. When comparing the revenue of the two games in 2015 , Clash of Clans came out ahead with $\$ 1.345$ billion while Game of Ware made $\$ 799$ million [4].

\section{Conclusion}

Revenue generation in F2P games is a challenging task for game providers due to heterogeneous consumers and strong competition from copycat games. In this study, we characterize optimal strategies for a monopoly game provider and for both the original and copycat game providers in a duopoly case.

In the duopoly model, we focus on popular F2P games with rich content. We show that there exists a pair of optimal solutions for the copycat provider's decision problem. To maximize revenue, both the optimal game challenge level and the optimal unit price for the virtual goods/currency should be set by copycat provider in observation of original game provider's strategy. It is also shown that there is an upper bound on the price of the copycat game provider, which regulates the unit price for virtual goods/currency set by copycat provider should be cheaper than that of the original game. Regarding the original game provider's strategy, we show that an optimal unit price for the virtual goods/currency does exist for the original game and the original game provider should set the challenge level of the game to the highest value possible to maximize its expected revenue anticipating copycat provider will cut its price in the duopoly setting. Moreover, we conclude that there should be a reasonable first mover advantage (in the form of discounted value to copycat games) for the original providers in order for them to create original games.

This study is not without its limitation. Although it integrates pricing decisions with F2P game design and heterogonous players, our model does not take players adaptive behaviors into consideration. Players may improve their playing skills hence modify their preferred game challenge levels as they become more skilled with gameplay mechanics, which in turn 
could affect game providers' strategies. Moreover, our model assumes the discount factor is the same for all players, but in actuality it might be different. In addition to this assumption, the duopoly case analysis is limited to popular F2P games with rich content. For future research, it will be interesting to expand the model to include players with multiple accounts for the same game and players playing both games in the duopoly case.

\section{References}

[1] Agarwal, R. and E. Karahanna. Time flies when you're having fun: Cognitive absorption and beliefs about information technology usage. MIS Quarterly, 24, 4 (2000), 665-694.

[2] App Annie \& IDC Portable Gaming Report 2014 Review. http:/go.appannie.com/app-annie-idc-portablegaming-report-2014-review/, Accessed on 2/2/2017.

[3] Cheng, H. K. and Liu, Y. Optimal software free trial strategy: the impact of network externalities and consumer uncertainty. Information Systems Research, 23, 2, (2012), 488-504.

[4] Crawley, D. Rise of the clones: why it pays (sometimes) to be a copycat mobile game.

https://www.chartboost.com/blog/2016/01/why-it-pays-tobe-copycat-mobile-game/, Accessed on 05/26/2016.

[5] Finneran, C. M. and P. Zhang. Flow in computermediated environments: Promises and challenges. Communications of the Association for Information Systems, 15, 1 (2005), 82-101.

[6] Global Games Market Report Infographics 2015. https://newzoo.com/insights/articles/global-games-marketreaches-99-6-billion-2016-mobile-generating-37/, Accessed on $08 / 20 / 2017$.

[7] Guo, H., Hao, L., Mukhopadhyay, T., \& Sun, D. Selling virtual currency in digital games: implications on gameplay and social welfare. In Theory in Economics of Information Systems, Information Systems Society 2015 pp. 1-28.

[8] Guo, Y. and Barnes, S. Why people buy virtual items in virtual worlds with real money. ACM SIGMIS Database, 38, 4 (2007), 69-76.

[9] Hamari, J. Why do people buy virtual goods? Attitude toward virtual good purchases versus game enjoyment. International Journal of Information Management, 35, 3 (2015), 299-308.

[10] Hamari, J. and Lehdonvirta, V. Game design as marketing: How game mechanics create demand for virtual goods. International Journal of Business Science \& Applied Management, 5, 1(2010), 14-29.

[11] Kearl, M. 30 Essential Stats on in-app Purchases and Monetization. https://www.appboy.com/blog/in-apppurchase-stats/, Accessed on 02/01/2017.

[12] Keating, L. Gaming on-the-go: the future of mobile gaming vs. consoles.

http://www.techtimes.com/articles/57048/20150604/gamin g-go-future-mobile-vs-consoles.htm, Accessed on $05 / 26 / 2016$.
[13] Liu, D., Li, X., and Santhanam, R. Digital games and beyond: What happens when players compete? MIS Quarterly, 37, 1 (2013), 111-124.

[14] Moon, J., Hossain, Md. D., Sanders, G. L., Garrity, E. J., and Jo, S. Player commitment to massively multiplayer online role-playing games (MMORPGs): an integrated Model. International Journal of Electronic Commerce, 17, 4 (2013), 7-38.

[15] Ryan, R. M., Rigby, C. S., and Przybylski, A. The motivational pull of video games: A self-determination theory approach. Motivation and Emotion, 30, 4 (2006), 344-360.

[16] Shapiro, C. Optimal pricing of Experience Goods, Bell Journal of economics, 14, 2 (1983), 497-507.

[17] Strauss, K. The \$2.4 Million-Per-Day Company: Supercell.

http://www.forbes.com/sites/karstenstrauss/2013/04/18/the2-4-million-per-day-company-supercell/ Accessed on 05/18/2016.

[18] Yee, N. Motivations for play in online games. CyberPsychology \& Behavior, 9, 6 (2006), 772-775. 